$(p=0.053)$ with a similar trend at 6 months. Baseline and 6 month SLEDAI means were higher in subjects with public compared to private insurance.

Baseline, 6 and 12 month SLEDAI means were significantly lower in Asian/PI compared to other ethnic groups. While White non-Hispanics demonstrated SLEDAI improvement over 6 months, the majority of Asians/PI, Blacks, and Hispanics demonstrated no change or worsened disease activity.

Conclusions Low socioeconomic status, as determined by the proxy variables of ethnicity and insurance type, is associated with greater mortality in SLE. To our knowledge, this is the first study that compares differences in treatment response in LN patients with low-SES in southern California. Our findings, which confirm the association of SES with long-term outcomes in SLE and LN, are in line with previous studies. More studies with greater power are warranted to validate these findings and improve healthcare outcomes.

Funding Source(s): None

\section{THE EFFECT OF HYDROXYCHLOROQUINE ON COMPLEMENT STATUS IN PATIENTS WITH SYSTEMIC LUPUS ERYTHEMATOSUS; ANALYSIS OF JAPANESE REAL-WORLD PATIENTS WITH SLE IN A LARGE SINGLE CENTER OVER TWELVE-MONTH PERIOD}

Yukihiko Ikeda*, Hiromichi Tamaki, Masato Okada. St Luke's International Hospital Immuno-Rheumatology Center

\subsection{6/lupus-2019-Ism.91}

Background Complement is a biomarker known to be correlate with disease activity of systemic lupus erythematosus (SLE). However, it is not well-known how complement level changes after starting hydroxychloroquine(HCQ), one of the key drugs for the treatment of SLE, in patients with SLE. The aim of this study is to investigate the effect of HCQ on complement levels over a 12 month period in a large single center cohort of SLE in Japan.

Methods We retrospectively collected the data of all the 244 lupus patients treated with HCQ from the electrical medical recordat St Lukes International Hospital, Tokyo, Japan. We extracted the following parameters during the period between April 2008 and March 2018; baseline characteristics,

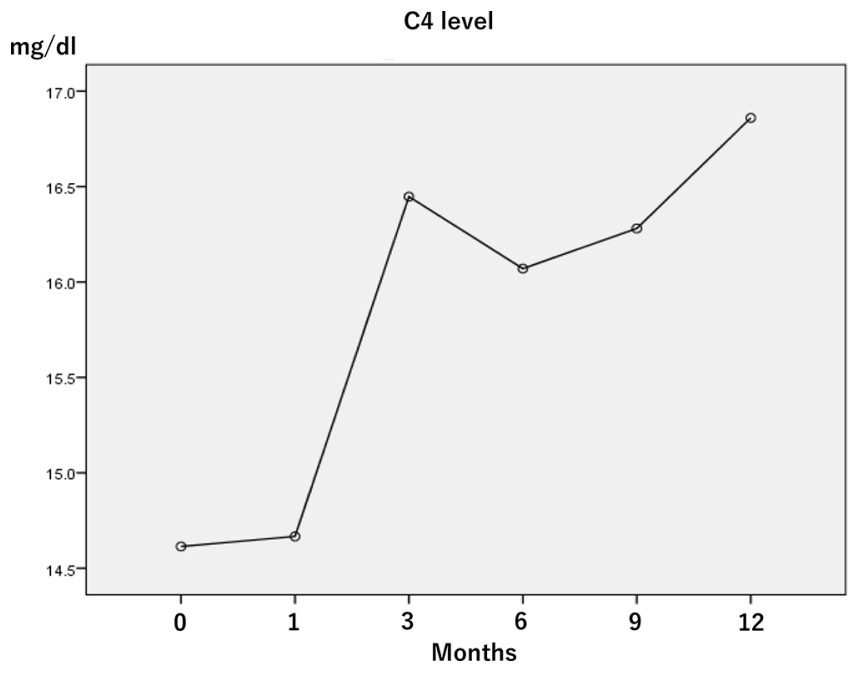

Abstract 91 Figure $1 \mathrm{C} 4$ level. The level of $\mathrm{C} 4(\mathrm{mg} / \mathrm{dL})$ increased significantly $(p<0.001)$ after starting HCQ complements levels (C3 and C4) at baseline1 month, 3 months, 6 months, 9 months, and 12 months after starting HCQ. Statistical analysis was performed using SPSS Statistics Version 21 (IBM Corp., Armonk, NY, USA). Mauchly's sphericity test and analysis of variance with Greenhouse-Geisser correction was used.

Results Total 244 patients on HCQ were included but 130 patients were excluded due to lack of sufficient data. The mean age of these 114 patients was 40.2 years and 108 patients $(94.7 \%)$ were female.

The level of $\mathrm{C} 4(\mathrm{mg} / \mathrm{dL})$ increased significantly $(\mathrm{p}<0.001)$ after starting HCQ. Especially, the level increased more dramatically from month 1 to month 3 compared to the other periods, and steadily increased event 6 months after initiating HCQ.. In terms of $\mathrm{C} 3$, it showed the same trend. The number of flares of disease activity has been calculated.

Conclusions The level of complements increases after initiating HCQ therapy in real-world patients with SLE in Japan. The effect tends to emerge 3 months after starting HCQ. We will show the data between disease activity and the elevation of complements.

Funding Source(s): None

\section{ENGAGING PATIENTS AND PARENTS TO IMPROVE MENTAL HEALTH FOR YOUTH WITH SYSTEMIC LUPUS ERYTHEMATOSUS}

${ }^{1}$ Andrea M Knight* ${ }^{*}{ }^{2}$ Oluwatunmise Fawole, ${ }^{3}$ Michelle Reed, ${ }^{4}$ Lauren Faust, ${ }^{5}$ Tamar Rubinstein, ${ }^{6}$ Julia Harris, ${ }^{7}$ Aimee Hersh, ${ }^{8}$ Karen Onel, ${ }^{9}$ Erica Lawson, ${ }^{10}$ Kaveh Ardalan, ${ }^{11}$ Esi Morgan, ${ }^{11}$ Anne Paul, ${ }^{12}$ Judith Barlin, ${ }^{13}$ Paola Daly, ${ }^{14}$ Mitali Dave, ${ }^{14}$ Shannon Malloy, ${ }^{14}$ Shari Hume, ${ }^{15}$ Suzanne Schrandt, ${ }^{14}$ Laura Marrow, ${ }^{3}$ Angela Chapson, ${ }^{3}$ Donna Napoli, ${ }^{3}$ Michael Napoli, ${ }^{3}$ Miranda Moyer, ${ }^{3}$ Rachel Adamski, ${ }^{16}$ Vincent Delgaizo, ${ }^{17}$ Martha Rodriguez, ${ }^{9}$ Emily von Scheven. ${ }^{1}$ The Hospital for Sick Children, Toronto; ${ }^{2}$ New York University School of Medicine; ${ }^{3}$ The Children's Hospital of Philadelphia; ${ }^{4}$ Philadelphia College of Osteopathic Medicine; ${ }^{5}$ Children's Hospital at Montefiore, New York, NY, USA; ${ }^{6}$ University of Missouri-Kansas City, Childrens Mercy-Kansas City, Kansas City, MO, USA; ${ }^{7}$ University of Utah, Salt Lake City, UT, USA; ${ }^{8}$ Hospital for Special Surgery, Weill Cornell Medicine, New York, NY, USA; ${ }^{9}$ University of California San Francisco, San Francisco, CA, USA; ${ }^{10}$ Lurie Childrens Hospital of Chicago, Chicago, IL, USA; ${ }^{11}$ Cincinnati Childrens Hospital Medical Center, Cincinnati, OH, USA; ${ }^{12}$ Lupus Foundation of America, Washington, DC, USA; ${ }^{13}$ Clinical Outcome Assessments; ${ }^{14}$ Cure $J M ;{ }^{15}$ Arthritis Foundation; ${ }^{16}$ Friends of CARRA; ${ }^{17}$ Riley Childrens Hospital at Indiana, Indianapolis, IN, USA

\subsection{6/lupus-2019-Ism.92}

Background Mental health conditions are common in youth with systemic lupus erythematosus (SLE), yet intervention strategies are understudied. We used a patient-engaged approach to investigate the mental health needs of youth with SLE.

Methods An anonymous online survey examined beliefs and experiences with mental health for youth with SLE. Eligible youth ages 14-24 years had a diagnosis of SLE and reported specific treatment for the condition. Parents of youth 8-24 years meeting the above criteria were also eligible to participate. The survey was developed in collaboration with patient and parent advisors, the Childhood Arthritis and Rheumatology Research Alliance (CARRA), and the Patients, Advocates, and Rheumatology Teams Network for Research and Service (PARTNERS). Participants were recruited through the Lupus Foundation of America and CARRA clinics. We tabulated youth responses for i) self-reported prevalence of mental health problems, categorized into mutually exclusive cliniciandiagnosed disorders and self-diagnosed symptoms, and ii) mean Likert ratings $(0=$ low, $4=$ high $)$ for the impact of disease related-factors on their mental health. We also compared 


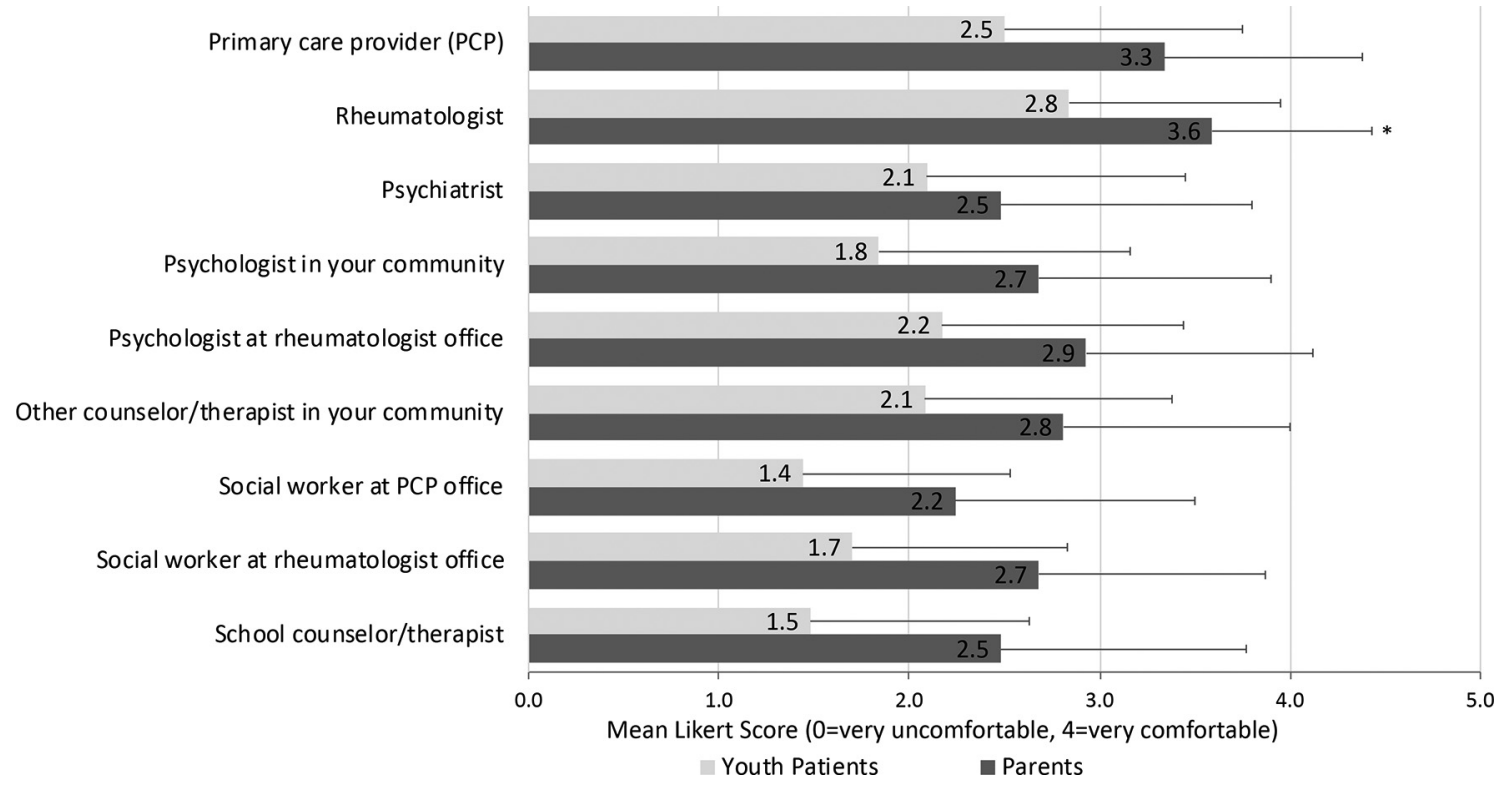

Abstract 92 Figure 1 Comparison of level of comfort with potential mental health providers among youth patients with SLE and parents. Results are shown for linear regression models comparing Likert scores among youth patients and parents, adjusted for disease duration and patient/parentreported visual assessment score for disease-related health. ${ }^{*}=p<0.05,{ }^{* *}=p<0.01,{ }^{* * *}=p<0.001$

youth and parent responses using regression models to examine comfort level with potential mental health providers.

Results 102 respondents included 59 patients (58\%) and 43 (42\%) parents. Youth had a mean age of 20.9 (standard deviation, $\mathrm{SD}=3.4$ ) years, and mean disease duration of 6.9 (SD 4.0) years. History of a mental health problem was reported by 21 youth (36\%), of which $66 \%$ said that their rheumatologist was unaware. Clinician-diagnosed anxiety was reported by $19 \%$, depression by $12 \%$, and adjustment disorders by $19 \%$; another $17 \%, 8 \%$ and $10 \%$ had self-reported symptoms of these disorders, respectively. Mean Likert ratings by youth indicated that disease aspects most impacting mental health were worry about disease impact on the future at 3.0 (SD 1.2), worry about having a flare at 2.9 (1.2), and worry about medication side effects at 2.8 (1.3). Youth and parents felt most comfortable discussing mental health concerns with rheumatologists and primary care providers, and least comfortable with social workers and school counselors (figure 1).

Conclusions Youth with SLE have high rates of diagnosed and undiagnosed mental health problems, which are impacted by their disease. Mental health intervention strategies in rheumatology settings may improve mental health education, screening and treatment for these youth.

Funding Source(s): The Childhood Arthritis and Rheumatology Research Alliance

\section{NEUROPSYCHOLOGICAL FACTORS AND ANTI- NEURONAL ANTIBODIES AS DAMAGE ACCRUAL PREDICTORS IN SYSTEMIC LUPUS ERYTHEMATOSUS}

\footnotetext{
${ }^{1}$ Milena Mimica, ${ }^{2}$ Ignacio Barra, ${ }^{2}$ Rocío Ormeño, ${ }^{2}$ Patricia Flores, ${ }^{2}$ Jorge Calderón, ${ }^{2}$ Oslando Padilla, ${ }^{1}$ Marcela Bravo-Zehnder, ${ }^{1}$ Loreto Massardo, ${ }^{3}$ Alfonso González. ${ }^{1}$ Centro de Biología Celular y Biomedicina (CEBICEM). Faculty of Science and Medicine, Universidad San Sebastián. Santiago, Chile; ${ }^{2}$ Faculty of Medicine, Pontificia Universidad Católica de Chile. Santiago, Chile; ${ }^{3}$ Centro de Biología Celular y Biomedicina. Faculty of Science and Medicine, Universidad San Sebastián. Santiago, Chile. Centro de Envejecimiento y Regeneración (CARE), Facultad de Ciencias, Pontificia Universidad Católica de Chile. Santiago, Chile
}

10.1136/lupus-2019-Ism.93
Background Organ damage may accumulate throughout life. The role of neuropsychiatric lupus manifestations on damage remains unknown. Cognitive dysfunction is present in $17 \%-66 \%$ of patients and an impact on damage accrual is suggested.We found a cognitive deficit association with the presence of anti-dsDNA/methyl-d-aspartate receptor (antidsDNA/NMDAR) and anti-ribosomal $\mathrm{P}$ proteins (anti-P), antibodies that have fluctuating titers with disease activity. Anti-P exerts neuropathogenic potential through recognition of neuronal surface antigens distributed in regions in memory, cognition and emotion. Anti-neuronal surface $\mathrm{P}$ antigen (NSPA), the anti-P target, is involved in neuronal excitatory transmission and mediates anti-P effects on memory dysfunction in mice models

We investigated predictors associated to damage in a SLE cohort with a thorough neuropsychological and antineuronal antibodies baseline assessment, after 4.8 years of follow-up. We include data on a new class of antibodies recognizing NSPA and lacking anti-P reactivity.

Methods From a study on 118 female SLE patients between 2008 and 2013 a subset of 99 SLE patients was followed-up in 2016. Baseline assessment: age, disease duration, therapies and antiphospholipid syndrome (APS). Neuropsychological evaluation: depression (MINI-plus) and cognitive deficit (Cambridge Neuropsychological Test Automated Battery). Antibodies: anti-dsDNA, anti-dsDNA/N-methyl-d-aspartate receptor (dsDNA/NMDAR), anti-P and anti-neuronal surface $\mathrm{P}$ antigen (NSPA). Baseline and last follow-up: activity index (SLEDAI2K), systemic damage index (SDI). Statistics: multivariate analyses.

Results At baseline: mean age (37.0 years; SD 12.7), disease duration (7.2 years; SD 8.2), SLEDAI-2K score (7.4; SD 6.0) and SDI score (0.8; SD 1.3). Major depression (23\%), cognitive deficit (18\%) and both (3.4\%). APS (9\%). Antimalarials (84\%) and cytotoxics (36\%). Anti-dsDNA (49\%), dsDNA/ NMDAR (19\%), anti-P (12\%) and anti-NSPA (5\%). After a mean follow-up of 4.8 (SD 2.0) years, $11.1 \%$ acquired new damage. Final SDI score was 1.0 (SD 1.6). In multivariate analysis baseline SDI, SLEDAI-2K and cytotoxics associated 